\title{
Homology bioinformatics analysis of IPA1 gene in higher plants
}

\author{
Zhongling Gong,Wenjie Xu \\ Gas group co., LTD of Shenzhen City $\cdot 518049 \cdot$ china
}

\begin{abstract}
IPA1 gene controlled paddy has increased in height, sturdy stem, number of tillers decreased, grains per panicle and dry grains weight increased significantly. In this study, the homology of IPA1 gene in 26 types of plant from 15 families was analyzed, and thus constructed its phylogenetic tree. The results of phylogenetic tree construction based on its amino acid sequence showed that the species from the same family, such as Gramineae, Rosaceae and Palmaceae, were well clustered for different branches and had high support rates. Furthermore, we constructed the phylogenetic tree based on its CDS sequence, and the species of Gramineae was well clustered, and the support rate was $100 \%$. Our results indicate that IPA1 has high homology in higher class of plants, especially in the Gramineae, which is of great significance for us to further study the yield of rice and other gramineous species.
\end{abstract}

Key words:IPA1 homology analysis sequence alignment phylogenetic tree

\section{Introduction}

Nearly half of the world's population take rice as the staple food, and more than 10,000 rice varieties provide a quarter of the global per capita dietary energy supply. Today, the world is facing that the land resource is declining, the world's population is increasing especially China's population. Therefore, it is increasingly important to improve the yield and quality of rice to meet the global population demand. In order to achieve the third breakthrough in paddy production, the existing varieties which are with high production are improved on the basis for higher production, high yield of paddy has become the world's popular research topic. The key to high yield of paddy is to have good plant morphology, and the ideal plant type is one of the main technical ways to cultivate super paddy [1].

In the early thirties, Boysen Jenson and Heath observed that the posture and number of leaves of plants could determine the differences in their material production, and proposed the concept of crop plant type. For the time being, the definition of plant type has not been identified as a uniform standard. Yang Shouren proposed to plant type is divided into group which are the general plant type (Plant Type), narrow plant type (Plant Morphology)[2]. In general, the plant type is referred to the pattern of the narrow plant which mainly refers to the shape of the leaf, the spatial arrangement of the leaves and the posture when receiving light. Plant type also includes some other constituent factors, namely root type, stem type and spike type.

There are some scholar carry out in-depth research on plant type in our country. The study of Ling Qihong and Lu Weiping showed that the distribution of roots is lighter, the smaller the number, the larger the leaf angle, the more scattered the leaves; the deeper the root distribution, the more of the number. The smaller the leaf angle, the more compact the leaves and easier for it to stay upright, it will be able to form a better group structure [3]. High-yielding paddy usually has a developed root system, and it is deep roots and peripheral roots [4] account for a larger proportion [5]. Ling Qihong that thick and strong stem can significantly improve the seed setting rate and weight of the thousand grain [6]. The spike of paddy was divided into three spike types, namely, erect panicle type, hemiplegic panicle type and curved panicle type [7]. The erect panicle type can best improve the population structure of the paddy group and its posture when absorbed the light. Studies have shown that the shading area of paddy is almost equal to the area index of a leaf, which affects the photosynthesis of the leaves, leading to a decrease in seed setting [7].

The formation of paddy plant type depends on plant height, tiller number and tiller angle [8]. The whole genome sequencing of gramineous plants such as paddy [9], maize [10] and sorghum [11] has been completed, together with the continuous research and progress of the structure and function of IPA1 gene. In order to select high cultivate quality, the new crops with high yield and nutrition rich has great significance. Due to the continuous development and progress of science and technology, scientists have combined the molecular biology and the physiological relationship of paddy plant type, and through the regulation of certain genes to change the external characteristics of paddy plants, thus the ideal plant type of paddy is selected.

The IPA1 gene, which controls the ideal plant type of paddy, was isolated from the ideal plant type "less tiller japonica", which was established by the Lijiayang Research Group of the Chinese Academy of Sciences, Institute of Genetics and Genetics, Chinese Academy of Sciences. The gene is a semi-dominant gene located on the 8th chromosome of paddy[12-14]. In order to make paddy yield to a new level, breeders proposed the idea of paddy ideal plant type. Through the cloning and analysis of the IPA1 gene, the investigators found that the IPA1 gene could greatly alter the shape of the plant and increase the yield. IPA1 encodes OsSPL14, a transcription factor containing SBP-box, which has the highest homology with Arabidopsis thaliana SQUAMOSA promoter binding protein LIKE-9 (SPL9) (At2g42200). IPA1 is localized in the nucleus and has transcriptional activation activity. The in situ expression analysis of the mRNA showed that OsSPL14 could control the tiller in the vegetative stage. The high expression of OsSPL14 could promote the spike branch in the reproductive stage.

The IPA1 gene of japonica paddy is $7229 \mathrm{bp}$ long, and its mRNA consists of three parts: 995 to 1566,397 to 4130,4233 to 5150; its complete CDS also includes three parts: 1118 to 1566,397 to 4130 , 4233 to 4903, respectively; IPA1 gene contains miR156 target site, miR156 can be regulated by transcription and translation inhibition. Because of the mutations in the target site of miR156, the effects of IPA1 transcripts and protein levels were also increased. The results of the transgenic study of IPA1 show that although mutations at the site lead to changes in amino acids, this mutation does not alter the 
2 Trends in Genetics and Evolution

function of the IPA1 protein. OsSPL14 mutation disrupts the regulation of OsmiRa56 on OsSPL14. Due to OsSPL14 mutations, the number of paddy tillers decrease, the number of grains per spike and weight of thousand grain increased, while the stems became thick, lodging resistance increased, and thus increased yield [9-10].

At present, the core areas of super paddy research at local and abroad are the breeding of ideal plant type. IPA1 controls the main quantitative traits of the ideal plant type of paddy. It encodes a transcription factor containing SBP-box and regulates multiple growth and development processes. The characteristics of mutants of IPA1 in paddy accorded with typical ideal plant type characteristics. IPA1 gene has great potential in the cultivation of super paddy cultivars. Therefore, homology analysis of IPA1 is of great significance not only to understand IPA1 more deeply, but also to use IPA1 in high-yield traits in other higher plants., in order to improve the production of crops in China and worldwide, to make a significant contribution to the development of agriculture around the world.

\section{1, Materials and methods}

1.1 Material source sequence

Using the online NCBI database to download the CDS and protein sequences of paddy IPA1, the CDS sequence number was GU136674.1 and the sequence of the protein sequence was ADJ19220.1. Therefore, the CDS sequence and the protein sequence were used as the target sequence to carry out the relevant homology bioinformatics analysis. The sequence to be analyzed is as follows: CDS sequence:

$>$ Oryza sativa Japonica Group IPA1 (IPA1) gene, cds

ATGGAGATGGCCAGTGGAGGAGGCGCCGCCGCCGCCG CCGGCGGCGGAGTAGGCGGCAGCGGCGGCGGTGGTGGTGG AGGGGACGAGCACCGCCAGCTGCACGGTCTCAAGTTCGGCA AGAAGATCTACTTCGAGGACGCCGCCGCGGCAGCAGGCGGC GGCGGCACTGGCAGTGGCAGTGGCAGCGCGAGCGCCGCGCC GCCGTCCTCGTCTTCCAAGGCGGCGGGTGGTGGACGCGGCG GAGGGGGCAAGAACAAGGGGAAGGGCGTGGCCGCGGCGGC GCCACCGCCGCCGCCGCCGCCGCCGCGGTGCCAGGTGGAGG GGTGCGGCGCGGATCTGAGCGGGATCAAGAACTACTACTGC CGCCACAAGGTGTGCTTCATGCATTCCAAGGCTCCCCGCGTC GTCGTCGCCGGCCTCGAGCAGCGCTTCTGCCAGCAGTGCAG CAGGTTCCACCTGCTGCCTGAATTTGACCAAGGAAAACGCA GCTGCCGCAGACGCCTTGCAGGTCATAATGAGCGCCGGAGG AGGCCGCAAACCCCTTTGGCATCACGCTACGGTCGACTAGCT GCATCTGTTGGTGAGCATCGCAGGTTCAGAAGCTTTACGTTG GATTTCTCCTACCCAAGGGTTCCAAGCAGCGTAAGGAATGCA TGGCCAGCAATTCAACCAGGCGATCGGATCTCCGGTGGTATC CAGT

GGCACAGGAACGTAGCTCCTCATGGTCACTCTAGTGCA GTGGCGGGATATGGTGCCAACACATACAGCGGCCAAGGTAG CTCTTCTTCAGGGCCACCGGTGTTCGCTGGCCCAAATCTCCC TCCAGGTGGATGTCTCGCAGGGGTCGGTGCCGCCACCGACT CGAGCTGTGCTCTCTCTCTTCTGTCAACCCAGCCATGGGATA CTACTACCCACAGTGCCGCTGCCAGCCACAACCAGGCTGCA GCCATGTCCACTACCACCAGCTTTGATGGCAATCCTGTGGCA CCCTCCGCCATGGCGGGTAGCTACATGGCACCAAGCCCCTGG ACAGGTTCTCGGGGCCATGAGGGTGGTGGTCGGAGCGTGGC GCACCAGCTACCACATGAAGTCTCACTTGATGAGGTGCACCC TGGTCCTAGCCATCATGCCCACTTCTCCGGTGAGCTTGAGCT TGCTCTGCAGGGGAACGGTCCAGCCCCAGCACCACGCATCG ATCCTGGGTCCGGCAGCACCTTCGACCAAACCAGCAACACG

\section{ATGGATTGGTCTCTGTAG}

Protein sequence:

> Gi | 299482812 | gb | ADJ19220.1 | IPA1 [Oryza sativa]

MEMASGGGAAAAAGGGVGGSGGGGGGGDEHRQLHGL KFGKKIYFEDAAAAAGGGGTGSGSGSASAAPPSSSSKAAGGGR GGGGKNKGKGVAAAAPPPPPPPPRCQVEGCGADLSGIKNYYC RHKVCFMHSKAPRVVVAGLEQRFCQQCSRFHLLPEFDQGKRSC RRRLAGHNERRRRPQTPLASRYGRLAASVGEHRRFRSFTLDFS YPRVPSSVRNAWPAIQPGDRISGGIQWHRNVAPHGHSSAVAGY GANTYSGQGSSSSGPPVFAGPNLPPGGCLAGVGAATDSSCALS LLSTQPWDTTTHSAAASHNQAAAMSTTTSFDGNPVAPSAMAG SYMAPSPWTGSRGHEGGGRSVAHQLPHEVSLDEVHPGPSHHA HFSGELELALQGNGPAPAPRIDPGSGSTFDQTSNTMDWSL

1.2 IPA1 gene homology bioinformatics analysis used by the software and operating procedures

1.2.1 Paddy homology analysis software and operating procedures

BLAST comparison on NCBI website;

Steps:

1, Open the NCBI, click BLAST, the page goes to the BLAST page;

2, Select the type of sequence to be homologous analysis, such as the nucleotide sequence click Nucleotide Blast, if the protein sequence is selected Protein Blast;

3 , In the page, copy and paste the analysis of the serial number or gene sequence, select the similarity of homology;

4, Click Blast, complete homology search;

5 , Refer to the value of $\mathrm{E}$ and $\mathrm{S}$, if $\mathrm{S}$ value is higher, the similarity is greater, E value as small as possible; download homology similar species of CDS and protein sequence.

1.2.2 IPA1 sequence alignment software and operating procedures

Sequencing using Bioedit;

Steps:

1, Download the sequence which needed to be compared was edited by using UltraEdit, that is, copy and paste into a text and save;

2, Use Bioedit to open the sequence which already been reedited;

3, Click Accessory Application, the emergence of a series of options, select ClustalW Multiple alignment;

4, Pop up a new window, based on the original choice Calculate NJ tree, FAST algorithm for bootstraps, Output Clustal format with Clustal consensus sequence generation;

5, Click Run ClustalW, click OK;

6, Result is showed, save, and analyze, and keep a copy.

1.2.3 Rice IPA1 system development tree construction software and operation steps

The phylogenetic tree was constructed by MEGA.

Steps:

1, With MEGA to open the software of the file of alignment sequence;

2, Then click Alignment, select Align by ClustalW;

3, Click to confirm;

4, Pop-up a new page, click Data;

5, Click Export Alignment, select MEGA Format;

6, The MEGA format file was open;

7, Click Phylogeny, select Bootstrap Test of Phylogcny, 
Construct / Test Neighbor-Joining Tree;

8, Click on Cumputer, NJ Tree showed;

9, Click File, select Export Current Tree (Newick), save NJ Tree.

\section{2, Results and analysis}

The number of amino acids in the IPA1 protein sequence was 513, there were 70 conserved loci, 411 mutations and 343 sites that provide information (Table 1).

Table 1 IPA1 amino acid sequence alignment results statistics

Number of amino acid Number of conserved loci

Number of mutation

variable Number of

parsim-info

$\begin{array}{llll}513 & 70 & 411 & 343\end{array}$

IPA1 coding sequence of the base number of 1391, the conserved locus is only 7 , the number of mutation sites is 1256 , can provide information on the site of 1199 (Table 2).

Table 2 Comparison of IPA1 CDS sequence comparison results Number of bases

nucleotide Number of Conservative Locations number of mutations

variable Number of

parsim-info

$\begin{array}{llll}1391 & 7 & 1256 & 1199\end{array}$

\subsection{IPA1 protein sequence homology analysis}

\subsubsection{Homology search of the IPA1 protein sequence}

The protein sequence of the protein encoded by paddy IPA1 gene in NCBI was ADJ19220.1, and then the IPA1 amino acid sequence of 31 other plants was retrieved by online Blast software (Table 3). They all have SBP conserved areas, including gramineous plants are sorghum, spike short stipe grass, section wheat, shortcuts wild rice, barley, millet, wheat, corn eight. In the IPA1 amino acid sequence of these Gramineae plants, there are knots of wheat, wheat, millet, corn and spikelet, and sorghum, maize, millet, short-seeded wild rice, barley and bis Spike. Overall, the scores of 32 sequences retrieved from Blast are relatively high, with scores between 207 and 545 , corresponding to $\mathrm{E}$ values in between $7 \mathrm{e}-59$ to 0.0 ; the gramineous plants have higher scores, $\mathrm{E}$ value is lower, the score is between 337 and 545, and the $\mathrm{E}$ value is between 1e-104 and 0.0 , which indicates that the IPA1 protein of gramineous plants is similar to that of paddy, and the similarity of other species is low.

Table 3 NCBI download species statistics

\begin{tabular}{|c|c|c|c|c|}
\hline Species name & \multicolumn{2}{|c|}{ Latin name } & Protein & Family \\
\hline Barley & Hondeum vulgare & 14 & \multicolumn{2}{|c|}{ Gramineae } \\
\hline paddy & \multicolumn{2}{|c|}{ Oryza sativa Japonica } & 14 & \\
\hline Spike Spicy & \multicolumn{2}{|c|}{ Brachypodium distachyon } & & \\
\hline Herbs & \multicolumn{2}{|c|}{ Oryza brachyantha 14 和 17} & \multicolumn{2}{|c|}{ Gramineae } \\
\hline ops & Aegilops tauschii & 17 & \multirow{2}{*}{\multicolumn{2}{|c|}{$\begin{array}{l}\text { Gramineae } \\
\text { Gramineae }\end{array}$}} \\
\hline & & & & \\
\hline Sorgh & Setar & 14 & \multicolumn{2}{|c|}{ Gramineae } \\
\hline Wheat & Triticum aestivum & 17 & \multicolumn{2}{|c|}{ Gramineae } \\
\hline White pear & \multicolumn{2}{|c|}{ Pyrus x bretschneideri } & 14 & Ros \\
\hline Apple & \multicolumn{2}{|c|}{ Malus domestica } & \multicolumn{2}{|c|}{ Rosaceae } \\
\hline Prunus & Prunus mume & 9 & \multicolumn{2}{|l|}{ Rosaceae } \\
\hline Wild Strawb & erry $\quad$ Fragaria & vesca & 9 & Rosacea \\
\hline
\end{tabular}

Sea Phoenix Phoenix dactylifera Branch

\begin{tabular}{lllll} 
Oil palm & Elaeis guineensis & 14 & \multicolumn{2}{c}{ Palm Branch } \\
Grape & Vitis vinifera & 9 & grape \\
Cucumber & Cucumis sativus & 9 & Cucurbitaceae \\
No oil camphor & Amborella trichopoda & 14 \\
\multicolumn{2}{l}{ Oil-free Lauraceae } & & &
\end{tabular}

Cocoa Tree Theobroma cacao 9

Cotton Gossypium arboreum

Indus Branch

Branch

Jatropha Jatropha curcas 9 Euphorbiaceae

Populus Populus euphratica $9 \quad$ Willow branch

Citrus Citrus trifoliata Rutaceae

Soybean Glycine max 9 Leguminosae

Lotus Nelumbo nucifera $17 \quad$ Water lily Branch

Circle Wensin $\quad$ Erycina pusilla 2 Orchid

Musa acuminata Musa acuminata 14 Banana family

\subsubsection{IPA1 protein sequence homology comparison}

The protein sequence of paddy was compared with the 31 amino acid sequences retrieved on Blast using ClustalW in Bioedit (Fig. 1). The results were shown at positions 57 to 67,139 to 228 , To the 360th, the 32 amino acid sequence has more of the same amino acids, indicating that these areas are conservative. Wherein, $141,143,144,147,148,152,154,157,158,159,160,161,164,166,170,172$, $174,178,179,180,181,182,183,184,185,181,189,191,192,193,196,198$, 199,200,202,203,204,205,206,207,208,209,210,211,245,350,353,360, 365 of the 50 amino acids are identical. It is noteworthy that in other areas, the 32 sequences do not have the same amino acid, reflecting the high variability of these regions.

Figure 1 Comparison of homology of rice IPA1 protein sequence

In the phylogenetic tree constructed with MEGA, two branches are given, and each branch will be described in detail below. The two branches from the top down were named branch $\mathrm{A}$ and branch $\mathrm{B}$.

In branch A, sorghum, corn, millet, rice, section wheat, wheat, barley, spikelet and these plants are the same group. Among them, the binding of protein 14 sorghum and corn into a sub-branch, the support rate of $98 \%$. This sub-branch together with the binding protein 14 millet together, the support rate of $93 \%$. And the subspecies of japonica paddy and the short-lived wild rice were the same. The support rate was $100 \%$. The control wheat was the same as that of wheat. The support rate was $100 \%$ and the barley with the binding protein 14 together, the support rate of $91 \%$; spike short stipe grass and the sub-branch together to form a new sub-branch, the support rate of $63 \%$; binding protein 17 millet, corn gathers, the support rate was $89 \%$; and with the binding protein 17 of the rice constitute as sub-branch, the support rate of $31 \%$; and then with the binding protein 17 spike short handle grass constitute a new sub-branch, the support rate of $40 \%$. Palm, including the sea phoenix, oil palm, they gets together, the support rate of $99 \%$, they and the banana family Basho subspecies constitute sub-support, support rate of $72 \%$. Gramineae, palmaceae, orchidaceae and orchid fan composition of sub-branch, but the support rate is not high, $40 \%$. The combination of protein 17 and the above sub-branches constitute a new sub-branch, the support rate of $81 \%$.

In Branches $\mathrm{B}$, the white pear and apples of Rosaceae were clustered together with a support rate of $82 \%$. They were associated 
with $89 \%$ of the metamorphosis of the protein 9 , which belonged to Rosaceae, and $89 \%$ at the same time. Of the wild strawberries together, get $91 \%$ support. They form a sub-branch with the same combination of protein 9, resulting in a low support rate of only $14 \%$. The cucumber combined with protein 9 is a sub-branch of the oil-bearing camphor, but the support rate is relatively low. The binding rate of the cocoa tree and the mallow tree of the Indus Branch of the protein 9 is $87 \%$, and the support is $87 \%$ Euphorbiaceae Lepidoptera constitute a new sub-branch, the support rate of $45 \%$; Populus euphratica Populus and Rutaceae Branch together, get the support rate of $45 \%$, this subbranch and the formation of a sub-branch The new sub-branch, 71\% support rate; support for the formation of sub-support with soybean $50 \%$; and water lily Branch of the lotus received by the support of $81 \%$.

From the phylogenetic tree, we can see that the plants that bind to protein 14 are clustered together, and the plants that bind to protein 17 are clustered together, and the plants that bind to protein 9 are clustered together and have a higher support rate.

Figure 2 Rice IPA1 protein sequence homology analysis NJ tree

2.2 IPA1 coding sequence homology analysis

2.2.1 Homology search for IPA1 coding sequence

The coding sequence was searched for SEQ ID NO: GU136674.1 according to the genetic sequence of paddy IPA. The IPA1 coding sequence of 14 other plants was retrieved by online Blast software (Table 2). They all have SBP conserved areas, including corn, millet, shortcuts wild rice, spikelets, barley, wheat, sorghum, oil palm, sea and banana. The score is between 1242 and 60.2, corresponding to an $\mathrm{E}$ value between $8 \mathrm{e}$ and 05 to 0.0 .

2.2.2 IPA1 coding sequence homology comparison

The CDS sequence for paddy using ClustalW in Bioedit was compared to the amino acid sequence retrieved on Blast (Figure 3). The results were shown at positions 125 to 158,356 to 988,129 , the 15 amino acid sequence has more of the same amino acids, indicating that these areas are conservative; of which 356 to 372,466 to 469,475 to 501,511 . Positions 521,553 to 572,646 to 658,952 to 988 , and the 1253 to 1284 amino acids are substantially identical, and these areas may be conserved. It is noteworthy that in other areas, the 15 sequences do not have the same amino acid, reflecting the high variability of these regions.

Figure 3 Comparison of homology of rice IPA1 coding sequence

There are two branches in the phylogenetic tree of the coding gene constructed with MEGA, and the two branches will be described in detail (Fig. 4). Now these two branches from top to bottom named branch one and branch two.

The branching consist mainly of gramineous plants, and the protein 17 , which binds to the protein 17 , is clustered with the small protein of the binding protein 17 at a support rate of $100 \%$; the shortlabeled wild paddy of the binding protein 17 is clustered together with the spikelet of the binding protein 17, the support rate of $100 \%$; from the phylogenetic tree can be seen that the majority of plants combined with protein 17 together, the support rate of up to $100 \%$; barley and the binding protein 17 wheat together, the support rate of $100 \%$, this sub
The support rate was $100 \%$, and this sub-branch formed a new subbranch with the previous sub-branch, but the support rate was low, only $38 \%$. Rice two subspecies japonica rice and short-lived wild rice together, the support rate of up to $100 \%$, combined with protein 14 corn and sorghum together, and with the binding protein 14 millet formation sub-support, the support rate is $100 \%$; the support rate is only $59 \%$ for the sub- branch from previous sub-branch into new branch.

In the branch two of the binding protein 17, the sea phoenix and the binding protein 14 of the oil palm together, the support rate of $90 \%$, and they belong to the palm family; this sub-branch and the binding protein 14 of the banana family of banana subspecies to form a new Of the sub- branch, its support rate of $100 \%$.

Figure 4 IPA1 coding gene homology analysis $\mathrm{NJ}$ tree

\section{3, Discussion and conclusions}

The IPA1 gene is a high-yielding gene of paddy which regulates the number of primary branches and participates in the regulation of the germination and branching stages of the vegetative growth stage. It can increase the function of panicle in the young stage. The study of IPA1 mainly focuses on its molecular mechanism and the mechanism of action.

In this study, the homology of IPA1 gene in higher plants was explored. The results showed that IPA1 gene was found in gramineous plants such as sorghum, maize, millet, rice, wheat, wheat, barley. The homology of IPA1 gene in paddy and some non-gramineous plants is relatively high, such as palms, and the distribution of IPA1 gene is relatively high in the phylogenetic tree, Tree oil palm, sea phoenix, rose family, apples, plum, wild strawberries, and cotton, soybeans and other crops. This also means that IPA1 may also play a role in increasing yields in other higher plants, especially in plants and crops that are closely linked to human life. This study will provide a basis for further understanding the biological function of IPA1 in higher plants. The expression mechanism and specific functional characteristics of IPA1 in other higher plants need further scientific experiments and research.

\section{REFERENCES}

[1] Zhang Lihua, Wang Mee. Advances in ideal plant type of super highyielding rice $[\mathrm{J}]$. Seeds, 2001, (5): 36-38.

[2] Chen Wenfu, Xu Zhengjin, Zhang Longjie. Physiological basis of super high-yielding breeding of rice [M]. Shenyang: Liaoning Science and Technology Press, 1995,21 (4): 213-217.

[3] Ling Qihong, Lu Weiping.Studies on the Relationship between Root Distribution and Leaf Corner of Rice [J]. Acta Agronomica Sinica, 1989,15 (2): 123-131

[4] Kosala R, Ernst S, Renee L. Control of water uptake by rice (Oryza sativa L.): Role of the outer part the root [J]. Planta.2003,217 (2): 193-205.

[5] Li Hesong, Huang Shiliang.Studies on root characteristics of doublecropping rice under super high-yielding cultivation [J] .Journal of Laser Biology, 1999,8 (3): 199-200.

[6] Ling Qihong.Study on the relationship between the number of vascular bundles and the panicle traits of rice stem [J]. Journal of Jiangsu Agricultural Sciences, 1982, (3): 48-58

[7] Ye Xinfu. Study on the Characters of Rice Plant Type [J]. Fujian Rice and Wheat Science and Technology, 1998,16 (2): 3-5.

[8] Wei Sha, Wu Jing, Zhang Shuangxi.Studies on crop plant-related genes

[J] .National and Fishery Technology, 2012,53 (4): 4-7,11

[9] Yu J, Wang GK, Li S, Liu B, Deng y, Dai L, Yang H. A draft sequence of the 
rice genome (Oryza sativa L.SSp.indica) [J]. Science, 2002,296 5): 79-92.

[10] Schnable PS, Doreen W, Robert S, Shiran P, Zhang JW, Kristi C, Scott JE, Richard K W. The B73 maize genome: complexity, diversity, and dynamics [J]. Science, 2009, 326 (5956): 1112-1115.

[11] Andrew HP, John EB, Remy B, Inna D, Jane G, Georg H, Therese M, Thomas W, Udo G, Apurva N, Bryan WP, Zhang LF, Doreen W. The Sorghum bicolor genome and the diversification of Grasses [J]. Nature, 2009,457 (7229): 551-556.

[12] YONGQING J, YONGHONG W, DAWEI X, et al. Regulation of OsSPL14 by Osmir156 defines ideal plant architecture in rice [J]. Nature genetics, 2010,42 (6): 541-544.

[13] KOTARO MIURA, MAYUKO IKEDA, ATSUSHI MATSUBARA, et al. OsSPI14 promotes panicle branching and higher grain productivity in rice [J]. Nature genetics, 2010,42 (6): 545-549. 